\title{
Peering into the heart of a high-mass star forming region: Bispectrum speckle interferometry of the ultracompact H II region K3-50 A*
}

\author{
K.-H. Hofmann ${ }^{1}$, Y. Y. Balega ${ }^{2}$, T. Preibisch ${ }^{1}$, and G. Weigelt ${ }^{1}$ \\ 1 Max-Planck-Institut für Radioastronomie, Auf dem Hügel 69, 53121 Bonn, Germany \\ 2 Special Astrophysical Observatory, Nizhnij Arkhyz, Zelenchuk region, Karachai-Cherkesia 357147, Russia
}

Received 5 November 2003 / Accepted 24 December 2003

\begin{abstract}
We present $K^{\prime}$-band bispectrum speckle interferometry of the ultracompact H II region K3-50A. Our image resolves the central $1^{\prime \prime} \times 1^{\prime \prime}$ region into at least 7 point-like objects. We find $K^{\prime}$-band counterparts for all but one of the $N$-band sources discovered by Okamoto et al. (2003), and there are additional $K^{\prime}$-band sources which were unresolved in the $N$-band images. Our reconstructed image also reveals the fine-structure of the cone-shaped nebulosity extending to the south. The brightest $K^{\prime}$-band source is located exactly at the tip of the cone-shaped nebulosity. The nebula shows several arcs and the orientation of its main axis agrees very well with the direction of the CO outflow from K3-50A. This nebulosity therefore very likely represents the clumpy inner surface of a partially evacuated cavity excavated by the strong outflows.
\end{abstract}

Key words. techniques: interferometric - stars: formation - stars: pre-main sequence -

H II regions: individual: G70.293+1.600

\section{Introduction}

The formation of massive stars is still a poorly understood process (cf. Garay \& Lizano 1999 for a recent review). One reason is that high-mass stars generally do not form in isolation but rather in the centers of dense clusters, which often display very crowded and confused morphologies. Another reason is that the evolutionary time scales of high-mass stars are very short. Massive stars start to burn hydrogen while still accreting material from the surrounding protostellar cloud. Very soon after the stellar core has formed, they vigorously affect their environment by strong UV radiation, powerful winds, and strong (molecular) outflows and jets. The UV radiation ionizes its surroundings and gives rise to an ultracompact $\mathrm{H}$ II region.

Due to the complexity of the environment in which highmass stars form, high spatial resolution imaging is required to reveal the morphological details. Our bispectrum speckle interferometry observations of several massive and intermediatemass young stellar objects have revealed very complex morphologies of their outflow structures, e.g. in S140 IRS 1 (Schertl et al. 2000; Weigelt et al. 2002a), AFGL 2591 (Preibisch et al. 2003), R Mon (Weigelt et al. 2002b), and have resolved many of the infrared sources into several components. e.g. S140 IRS 3 (Preibisch et al. 2001) and Mon R2 IRS 3

Send offprint requests to: T. Preibisch, e-mail: preib@mpifr-bonn.mpg.de

* Based on observations obtained at the Special Astrophysical Observatory with the $6 \mathrm{~m}$ telescope.
(Preibisch et al. 2002). This can give new insight into the distribution and physical properties of dusty circumstellar matter and the behavior of outflows close to their launching sites.

Due to its brightness in the near-infrared, the K3-50 A region is a very suitable target for a high spatial resolution study of a site of massive star formation.

$\mathrm{K} 3-50$ is an ultracompact $\mathrm{H}$ II region at a distance of $8.7 \mathrm{kpc}$ (Harris 1995). The region consists of four components (A-D; Wynn-Williams 1969) at different stages of star formation, among which K3-50 A (alias G70.293+1.600) seems to be the youngest component. At optical wavelengths the region is dominated by the $\mathrm{H}$ II region K3-50 D. At infrared wavelengths, however, the most prominent feature is the bright emission at the position of K3-50 A, which is very faint at optical wavelengths. Near-infrared images (see, e.g., Hodapp 1994) show the whole area filled by diffuse emission, connecting all four H II regions. Kurtz et al. (1994) determined the infrared luminosity of K3-50 A to be $2.28 \times 10^{6} L_{\odot}$. The region is embedded in a torus of molecular gas with a total mass of $\sim 2000 M_{\odot}$ (Vogel \& Welch 1983). Radio observations showed a very compact structure with a size of only $\sim 2$ " (Turner \& Matthews 1984). CO radio observations also suggested the presence of a bipolar outflow that is viewed nearly pole-on (Phillips \& Mampaso 1991). Radio observations by DePree et al. (1994) revealed a high-velocity bipolar outflow of ionized gas from K3-50 A. They found a pronounced velocity gradient of $\sim 150 \mathrm{~km} \mathrm{~s}^{-1} \mathrm{pc}^{-1}$ along the major axis at $\mathrm{PA}=160^{\circ}$. 
Howard et al. (1996) presented (seeing-limited) nearinfrared images of the K3-50 region. They found that the overall structure of K3-50 A appears to be that of a rotating torus of dense gas with a bipolar ionized outflow breaking through to the north and south. They derived an extinction of $A_{\mathrm{V}} \gtrsim 30 \mathrm{mag}$ towards K3-50 A and speculated that the formation of K3-50 A may have been induced by a recent supernova explosion. Radio observations of K3-50 A by Howard et al. (1997) revealed a flattened cloud of dense molecular gas $\sim 1.1 \mathrm{pc}$ in extent and a $\sim 0.5 \mathrm{pc}$ inner torus. This torus surrounds a bright continuum source, which is associated with the origin of the bipolar ionized gas outflow. The axis of the bipolar flow of ionized gas is perpendicular to the major axis of the molecular torus.

Okamoto et al. (2003) presented $N$-band imaging and spectroscopy and near-infrared images of K3-50 A. Their images have a spatial resolution of $0.4^{\prime \prime}$, but the emission peak in their $K$-band image is saturated, leaving the central $\sim 1^{\prime \prime}$ region unresolved. The $N$-band emission is strongly concentrated into a bright core with a size of $1.5^{\prime \prime} \times 1.5^{\prime \prime}$. The spatial structure of the emission in this core seems to suggest multiple components of emission, but they could not exclude the possibility that the inhomogeneities are just caused by fluctuations in the density distribution in the region. Okamoto et al. (2003) concluded that the core probably consists of at least three independent $N$-band sources within $1^{\prime \prime}$ from the emission peak, which they called OKYM 1, OKYM 2, and OKYM 3. However, the suggested sources OKYM 2 and OKYM 3, separated by only $0.4^{\prime \prime}$ were not clearly resolved in the $N$-band data. Another emission peak, called OKYM 4, was found 2" south-east of OKYM 3, and a further likely source, which they called STHO 1, was found $\sim 0.7^{\prime \prime}$ south-west of OKYM 3. They identified the peak in the $K$-band image with their $N$-band source OKYM 3 and also found a $K$-band counterpart for OKYM 4.

In the past, it had been assumed that the central source of K3-50 A (and other ultracompact $\mathrm{H}$ II regions in general) was a single massive protostar. The observations of Okamoto et al. (2003), however, put this assumption into question: they found a remarkable discrepancy in the Lyman continuum flux from K3-50 A derived from radio observations, which corresponds to the expected output of a single 05.5V star, and the mid-infrared line-flux ratios, which suggest much later spectral types (O8-B0) for the ionizing stars. Okamoto et al. (2003) therefore proposed that K3-50 A is excited by at least two, possibly three ionizing stars, and regarded this as an example of a massive stellar cluster as the ionizing source of an ultracompact $\mathrm{H}$ II region. The ionizing stars are OKYM 3 (or an invisible object $0.2^{\prime \prime}-0.3^{\prime \prime}$ to the north of OKYM 3), OKYM 4 , and STHO 1. The estimated spectral types of these objects are O7-O9, O9-B0, and O9-B0, respectively.

An at least qualitatively similar result was obtained by Lumsden et al. (2003): they compared the radio Lyman continuum flux and the far-infrared luminosity of K3-50 A and found that these quantities cannot be reproduced by the action of a single star, but require the presence of a cluster of several massive stars.

To obtain greater insight into the structure of this region and the nature of the central source in K3-50 A, observations with better spatial resolution are clearly needed. The aim of our high-resolution imaging study in the $K$-band presented here was to reveal more details of the innermost structure in this complex and interesting region.

\section{Observations and data analysis}

The speckle interferograms of K3-50 A were recorded on 24 September 2002 with the SAO 6 m telescope in Russia. The detector of our speckle camera was a Rockwell HAWAII array (only one $512 \times 512$ quadrant was used). The size of one pixel corresponds to 27.0 mas on the sky. A $K^{\prime}$-band filter with central wavelength $2115 \mathrm{~nm}$ and bandwidth $214 \mathrm{~nm}$ was used. The simultaneously recorded speckle interferograms of an unresolved star close to K3-50 A (distance 10") were used for the calculation of the atmospheric speckle transfer function. The exposure time per frame was $300 \mathrm{~ms}$. Our data set consists of 980 speckle interferograms of K3-50 A and of the unresolved reference star. The seeing was $\sim 1.1^{\prime \prime}(F W H M)$. The modulus of the Fourier transform of the object (visibility) was obtained with the speckle interferometry method (Labeyrie 1970). An image with a resolution of 106 mas (Fig. 1, top) was reconstructed using the bispectrum speckle interferometry method (Weigelt 1977; Weigelt \& Wirnitzer 1983; Lohman et al. 1983; Hofmann \& Weigelt 1986).

\section{Results}

Our speckle image shows a bright, point-like source at the northern tip of a cone-like nebula extending towards the south, in which a number of fainter, point-like sources are embedded. We will denote the point-like sources in our image by numbers 1 to 10 . The brightest source, number 1, appears point-like. A comparison of its spatial profile with the profiles of other point sources in the image shows no indications for being extended.

Comparing our image with the $0.4^{\prime \prime}$ resolution seeinglimited $K$-band image presented by Okamoto et al. (2003; their Figs. 3 and 4) allows a reliable cross-identification of the sources. Our brightest source 1 corresponds to the brightness peak in the seeing-limited $K$-band image of Okamoto et al. (2003), and our sources 8 and 10 can be reliably identified with point-like sources in their image. The rest of our sources, i.e. numbers 2 to 7 and number 9 , are not resolved in the $K$-band images of Okamoto et al. (2003).

This cross-identification also allows us to search for counterparts of the $N$-band sources in our $K$-band speckle image. Since Okamoto et al. (2003) suggested that the brightest $K$-band source corresponds to the $10 \mu \mathrm{m}$ source OKYM 3 , we assume here that our source 1 is positionally coincident with OKYM 3. OKYM 2 can then be identified with our $K$-band source 3, OKYM 1 with $K$-band source 5, and OKYM 4 with $K$-band source 10 . Only for the $10 \mu \mathrm{m}$ source STHO 1 we can see no counterpart in our $K$-band image ${ }^{1}$. Our speckle image directly confirms the presence of multiple sources in the core

\footnotetext{
We note that a near-infrared counterpart for STHO 1 is seen in the (deeper) $J H K$ images of Okamoto et al. (2003); its $K$-band emission is probably too faint to be detected in our speckle data.
} 

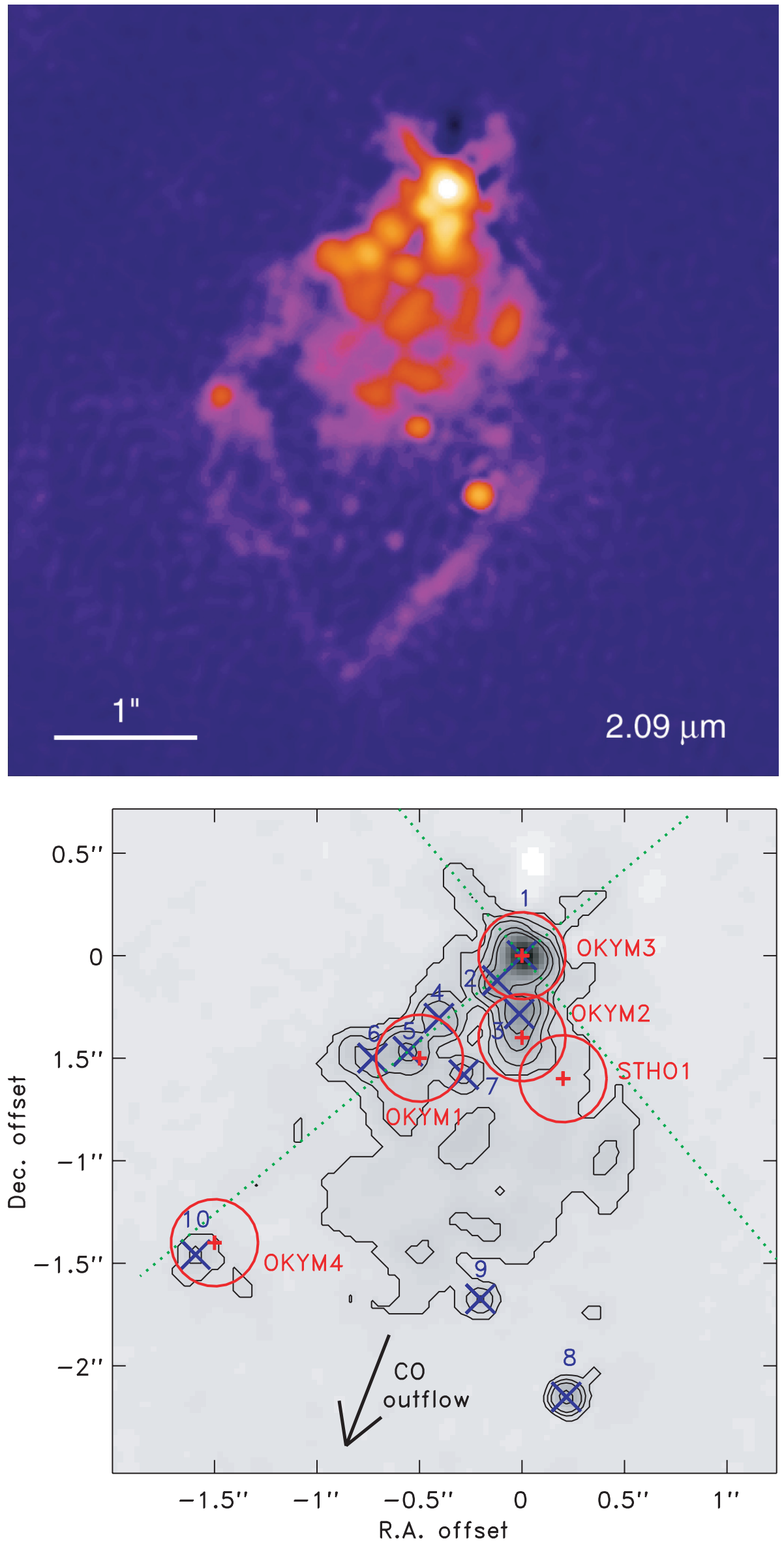

Fig. 1. Pseudocolor representation of our $K^{\prime}$-band image of K3-50 A, reconstructed with the bispectrum speckle interferometry method. This image has a resolution of 106 mas, the field of view is $\sim 5.4^{\prime \prime} \times 5.4^{\prime \prime}$. North is up and east is to the left.

Fig. 2. Greyscale representation of the central part of our $K$-band speckle image with annotation of the objects discussed in the text. The red crosses (+) mark the $10 \mu \mathrm{m}$ sources detected by Okamoto et al. (2003), the red circles give the positional uncertainty. The blue crosses $(\mathrm{X})$ mark the pointlike sources in our $K$-band speckle image for which we performed aperture photometry. The direction of the $\mathrm{CO}$ outflow is indicated by the arrow. 
Table 1. Relative positions and photometry for the point like sources in our $K^{\prime}$-band bispectrum speckle image. The J2000 coordinates of source 1 determined from the 2MASS $K$-band image are RA 20:01:45.7, Dec $\sim+33: 32: 44$.

\begin{tabular}{lrrrcc}
\hline \hline $\begin{array}{l}\text { Source } \\
\text { number }\end{array}$ & $\begin{array}{r}\Delta \mathrm{RA} \\
{[\mathrm{mas}]}\end{array}$ & $\begin{array}{r}\Delta \text { Dec } \\
{[\mathrm{mas}]}\end{array}$ & $\begin{array}{r}\Delta K^{\prime} \\
{[\mathrm{mag}]}\end{array}$ & $\begin{array}{c}\text { Identification with Okamoto et al. (2003) } \\
K \text {-band }\end{array}$ & $N$-band \\
\hline 1 & 0 & 0 & 0 & yes & OKYM 3 \\
2 & 135 & -108 & 1.74 & saturated/unresolved & OKYM 2 \\
3 & 13 & -256 & 1.65 & saturated/unresolved & \\
4 & 405 & -283 & 3.13 & unresolved & \\
5 & 567 & -445 & 2.83 & unresolved & OKYM 1 \\
6 & 742 & -513 & 3.36 & unresolved & \\
7 & 297 & -567 & 3.84 & unresolved & \\
8 & -216 & -2146 & 2.61 & yes & \\
9 & 202 & -1674 & 3.65 & $?$ & OKYM 4 \\
10 & 1593 & -1431 & 4.08 & yes & \\
\hline
\end{tabular}

of K3-50 A, and also confirms the indication from the $N$-band data that OKYM 2 and OKYM 3 are separate sources.

We performed aperture photometry for the 10 point-like sources in our speckle image with 40 mas aperture radii. The positions, relative magnitudes, and identifications of our $K^{\prime}$-band sources are listed in Table 1 . If we add up the fluxes of all 10 point-like sources, we find that source 1 contributes $58 \%$ and sources $2-10$ contribute $42 \%$ of the summed $K^{\prime}$-band flux. The $K^{\prime}$-band flux from the second brightest object, source 3 , is about 4.5 times weaker than that of source 1 . We conclude that the $K^{\prime}$-band emission is dominated by the brightest source 1 , but the other sources produce a significant fraction of the total emission.

We can also use the observed magnitude differences to check the suggested spectral types of the main sources in the K3-50 A core (Okamoto et al. 2003): if we assume that the relative $K^{\prime}$-band magnitudes of the sources are roughly representative of their relative luminosities, we find that the 4.5 times smaller flux of the second brightest source is consistent with a spectral type $\sim \mathrm{B} 0$ if the brightest source is of spectral type $\sim 08$. Therefore, the spectral type estimates of the ionizing sources in K3-50 A derived by Okamoto et al. (2003) are supported by our data.

It is also interesting to compare the $K^{\prime}$-band and $N$-band fluxes of the sources. Inspection of the $N$-band spectra (Okamoto et al. 2003) of OKYM 3 (our source 1) and OKYM 4 (our source 10) shows that these two sources have quite similar fluxes at $\sim 10 \mu \mathrm{m}$. In the $K^{\prime}$-band, however, OKYM 4 is more than 4 mag fainter than OKYM 3. If the intrinsic properties of both objects were identical, this difference in $K^{\prime}-N$ color would indicate that OKYM 4 suffers from about 140 mag more optical extinction than OKYM 3. Alternatively, OKYM 4 may also be surrounded by a much larger amount of warm ( $T \sim 500 \mathrm{~K}$ ) circumstellar material than OKYM 3, causing a strong mid-infrared excess.

This might suggest that source 10 is surrounded by a much larger amount of warm circumstellar material than source 1.

\section{The diffuse emission}

The diffuse nebulosity surrounding the point-like sources displays some quite remarkable features. The main axis of the diffuse cone-shaped nebulosity is $\mathrm{PA} \sim 175^{\circ}$ This agrees quite well with the position angle of $\mathrm{PA}=160^{\circ}$ that was found for the high-velocity bipolar radio outflow by DePree et al. (1994). The full opening angle is $90^{\circ}$. Other very interesting features are the straight edges of the diffuse nebulosity and the X-like structure around the brightest source. The direction of the short northern extensions corresponds well with the directions of the edges in the southern direction. This structure therefore seems to represents a well-defined cone with source 1 at its center.

These results suggest the following interpretation of the nebulosity: what we see is the clumpy inner surface of a partially evacuated cavity in the circumstellar envelope around the central source, which has probably been excavated by the strong outflow. The fact that the southern part of the cavity is much brighter than the northern part, suggests that the southern outflow structure is tilted towards the line-of-sight and that we therefore look "into" the southern cavity. The northern part of the bipolar cavity is much fainter because it is oriented away from us and, furthermore, is hidden by circumstellar extinction.

The outflow most likely originates from or is dominated by source 1 , because this is the brightest and therefore presumably most luminous object, and the cavity system is clearly centered on source 1 . The diffuse emission has a very clumpy structure with several prominent knots and some rather sharp and straight features. We note that the general morphology of the diffuse structures in our $K$-band speckle image is very similar to that seen in the $N$-band image of Okamoto et al. (2003).

One of the most peculiar features is the V-shaped structure at the southern end. The western part of the V-shaped structure corresponds to the northern part of "arc 1" defined by Okamoto et al. (2003). In our speckle image this part of the arc appears very sharp and remarkably straight.

It is also interesting to note that all the point-like $K$-band sources in our image are located within (or very close to the edges of) the southern cavity. As it seems quite unlikely that all members of a stellar cluster assemble in a cone-like volume and not in a roughly spherical distribution around the central source, this may imply that we can detect only those objects that are located in the cavity, because the extinction along the line-of-sight through the cavity is much lower than through other parts of the cloud. We suspect that the cluster contains more members, most of which, however, are hidden 
in the dense cloud material and are therefore not visible in our $K^{\prime}$-band speckle image.

The stronger extinction outside the cavity can, however, hardly explain the non-detection of further $\mathrm{N}$-band sources; if there were, e.g., further O-stars, the extinction would have to be extremely large to prevent their detection in the $N$-band. However, the sensitivity limit and the strong nebular emission in the $N$-band image allowed only the detection of the most massive objects, probably O-type and very early B-type stars. Slightly less massive cluster members are too faint to be detected in the $N$-band image.

\section{Summary and conclusions}

Our high-resolution imaging resolves the central $K^{\prime}$-band emission of K3-50 A into 10 point-like sources. The brightest $K^{\prime}$-band source dominates the near-infrared emission and, since it is centered on the bipolar cavity structure, it also seems to be the dominant driving source of the molecular outflow. However, the magnitudes of the other point-like sources indicate that some of them are also rather massive (probably early B-type) stars. This suggests that K3-50 A is not dominated by a single massive star, but by a small cluster of massive to intermediate-mass stars. Our results demonstrate the importance of high spatial resolution observations for revealing the true nature of massive YSOs.

Acknowledgements. This publication makes use of data products from the Two Micron All Sky Survey, which is a joint project of the University of Massachusetts and the Infrared Processing and Analysis Center, funded by the National Aeronautics and Space Administration and the National Science Foundation.

\section{References}

DePree, C. G., Goss, W. M., Palmer, P., \& Rubin, R. H. 1994, ApJ, 428,670

Harris, S. 1975, MNRAS, 170,139

Hodapp, K.-W. 1994, ApJS, 94, 615

Howard, E. M., Pipher, J. L., Forrest, W. J., \& De Pree, C. G. 1996, ApJ, 460, 744

Howard, E. M., Koerner, D. W., \& Pipher, J. L. 1997, ApJ, 477, 738

Kurtz, S., Churchwell, E., \& Wood, D. O. S. 1994, ApJS, 91, 659

Labeyrie, A. 1970, A\&A, 6, 85

Lohmann, A. W., Weigelt, G., \& Wirnitzer, B. 1983, Appl. Opt., 22, 4028

Lumsden, S. L., Puxley, P. J., Hoare, M. G., Moore, T. J. T., \& Ridge, N. A. 2003, MNRAS, 340, 799

Okamoto, Y. K., Kataza, H., Miyata, T., et al. 2003, ApJ, 584, 368

Phillips, J. P., \& Mampaso, A. 1991, A\&AS, 88, 189

Preibisch, Th., Balega, Y. Y., Schertl, D., Smith, M. D., \& Weigelt, G. 2001, A\&A, 378, 539

Preibisch, Th., Balega, Y., Schertl, D., \& Weigelt, G. 2002, A\&A, 392, 945

Preibisch, Th., Balega, Y., Schertl, D., \& Weigelt, G. 2003, A\&A, 412, 735

Schertl, D., Balega, Y., Hannemann, T., et al. 2000, A\&A, 361, L29

Thronson, H. A., \& Harper, D. A. 1979, ApJ, 230, 133

Turner, B. E., \& Matthews, H. E. 1984, ApJ, 277, 164

Vogel, S. N., \& Welch, W. J. 1983, ApJ, 269, 568

Weigelt, G. 1977, Opt. Commun., 21, 55

Weigelt, G. 1991, in Progress in Optics, 29, ed. E. Wolf (Elsevier Science Publishers), 295

Weigelt, G., Preibisch, Th., Schertl, D., Balega, Y. Y., \& Smith, M. D. 2002a, A\&A, 381, 905

Weigelt, G., Balega, Y., Hofmann, K.-H., \& Preibisch, Th. 2002b, A\&A, 392, 937

Wynn-Williams, C. G. 1969, ApL, 3, 195 